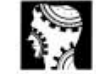

original papers
7 Department of Health. National Schedule of Reference Costs 2006-2007 for NHS Trusts. Department of Health, 2007.

8 HM Inspectorate of Prisons. Patient or Prisoner? A New Strategy for Healthcare in Prisons. Home Office, 1996.

9 Robertson G, Dell S, James K, Grounds A. Psychotic men remanded in custody to Brixton Prison. $\mathrm{Br}$. Psychiatry 1994; 164: 55-61.

10 Banerjee S, O'Neill-Byrne K, ExworthyT, ParrottJ. The Belmarsh
Scheme. A prospective study of the transfer of mentally disordered remand prisoners from prison to psychiatric units. BrJ Psychiatry 1995; 166: 802-5.

11 Isherwood S, Parrott J. Audit of transfers under the Mental Health Act from prison - the impact of organisational change. Psychiatr Bull 2002; 26: 368-70.

12 McKenzie N, Sales B. New procedures to cut delays in transfer of mentally ill prisoners to hospital. Psychiatr Bull 2008: 32: 20-2.
13 Wilson S, Forrester A. Too little, too late? The treatment of mentally incapacitated prisoners. J Forensic

14 Riviere v. France (2006) ECHR Application no. 33834/03, 11 July.

Psychiatry 2002: 13: 1-8.

*Andrew Forrester Consultant and Honorary Senior Lecturer in Forensic Psychiatry, Healthcare Department, HM Prison Brixton, Jebb Avenue, London SW2 5XF, email: andrew.forrester@ slam.nhs.uk, Christopher Henderson Formerly Research Associate, Bracton Centre Medium Secure Unit, Bexley, Simon Wilson Consultant and Honorary Senior Lecturer in Forensic Psychiatry, Oxleas NHS Foundation Trust and Institute of Psychiatry, London, lan Cumming Consultant in Forensic Psychiatry, HM Prison Belmarsh, London, Miriam Spyrou Assistant Psychologist, Bracton Centre Medium Secure Unit, Bexley, Janet Parrott Consultant in Forensic Psychiatry, Bracton Centre Medium Secure Unit, Bexley

\title{
How specialist ECT consultants inform patients about memory loss
}

\section{AIMS AND METHOD}

A questionnaire was distributed to consultants with a special interest in electroconvulsive therapy (ECT) at clinics participating in an ECT accreditation process. This aimed to ascertain a consensus of clinical practice regarding informing patients about the treatment and assessment of memory during ECT.

\section{RESULTS}

The response rate was $64 \%$. There is consensus on informing patients about the possibility of permanent memory loss. Memory is assessed before and during an ECT course by clinical interview and Mini-Mental State Examination, but rarely at longterm follow-up.

\begin{abstract}
CLINICAL IMPLICATIONS
Patients need to be informed about the possibility of permanent memory loss before consenting to ECT. Clinical teams need to make greater efforts to assess memory, particularly after this treatment.
\end{abstract}

Electroconvulsive therapy (ECT) is an effective short-term treatment for depression and is 'probably more effective than drug therapy'. However, the treatment remains controversial, with adverse effects being a major concern of both patients and the public, in particular the risk of memory impairment. A review of patients' perspectives on ECT found that at least a third of patients reported persistent memory loss after treatment. ${ }^{2}$

Research into the effect of ECT on memory function is a rapidly changing field. Previously, it was accepted that this treatment could cause temporary anterograde and retrograde amnesia, which could be minimised by using unilateral rather than bilateral ECT; ${ }^{3}$ however, current work is clarifying the possibility and nature of more persistent memory loss. A recent systematic review suggested that autobiographical memory impairment after ECT can occur, recovering after several months, although a few studies reported more persistent deficits. ${ }^{4}$ A large prospective study found that cognitive deficits, including deficits in autobiographical memory, were apparent 6 months after completion of a course of $\mathrm{ECT}^{5}$

There is concern that inadequate information is given to patients about the adverse effects of ECT, specifically with regard to the effect on memory. ${ }^{6,7}$ The ECT Accreditation Service (ECTAS) has previously studied the clinical practice and training needs of psychiatrists who refer patients for ECT. These referring psychiatrists were likely to have difficulty explaining to patients the possibility of long-term cognitive side-effects, with a spread of opinion among them, ranging from informing patients about long-term cognitive side-effects, to saying that there was no evidence of such side-effects, or saying that the issue is controversial. Just over a third recognised further training needs, particularly on consent and assessment of memory during and after ECT. ${ }^{8}$ There has been a gradual decline from 1985 to 2002 in the number of people in England receiving ECT, ${ }^{9}$ and it is likely that this trend has continued. Thus referring psychiatrists will have less experience of this treatment.

The aim of our study was to gain a greater understanding of the clinical practice of psychiatrists with responsibility for administration of ECT in the UK (ECT specialists or lead ECT clinicians, hereafter referred to as ECT consultant psychiatrists), with specific reference to what they tell patients about the effects of ECT on memory and how they approach detecting and monitoring these effects. This was in order to obtain a consensus of expert opinion in this area.

\section{Method}

The ECTAS was established in 2003 to improve the quality of ECT provision in England, Wales, Northern Ireland and Ireland. Clinics judged to provide a 
satisfactory service to patients are accredited by ECTAS. At the time of this study (in 2007) ECTAS had recruited 87 member clinics out of an estimated 149 clinics. ${ }^{10}$ One feature of signing up to the accreditation process is access to an email discussion forum. This forum was used for the purpose of recruitment in this study.

A questionnaire, consisting of 13 questions relating to issues about memory and ECT, was emailed to 108 practising UK psychiatrists identified from the discussion forum. The questionnaire included items requiring yes/no answers and open-ended questions where more detailed responses could be entered. Two further mailings were sent around 1 week and 7 weeks after the initial mailing. In addition, a written version of the questionnaire was sent by post $3-5$ months later.

Descriptive statistics were used to present the results. Not all respondents answered all questions. Responses to open-ended questions were analysed qualitatively, whereby responses were coded and grouped into themes. The authors then met to agree a final coding framework.

\section{Results}

A total of 108 questionnaires were distributed, resulting in 69 responses (a $64 \%$ response rate). All the respondents were consultant psychiatrists and 62 of the 69 identified themselves as ECT consultant psychiatrists. All

\section{Table 1. Responses to questions about the effect of ECT on memory}

Number giving

positive

response/total

answering

question $n / N$

$\%$

Do you discuss the possibility of memory loss with patients who you refer for ECT?

Do you inform them that

memory loss may be:

Temporary?

Permanent?

For events just before and after ECT?

For events long before ECT (retrograde amnesia)?

For events that occur more

than 3 weeks after ECT?

Are patients given a fact sheet

that includes information on

possible memory loss?

Do you give patients a choice

between unilateral and bilateral

ECT?

\begin{tabular}{lcc} 
Yes & $26 / 62$ & 42 \\
No & $6 / 62$ & 10 \\
Sometimes & $30 / 62$ & 48 \\
\hline
\end{tabular}

$E C T$, electroconvulsive therapy. the results presented below are from this latter subgroup, $90 \%$ of the whole sample, hereafter referred to as the respondents. The responses of the seven psychiatrists $(10 \%)$ who were not ECT consultant psychiatrists were excluded from further analysis. Of the 62 respondents, 33 (53\%) were general adult psychiatrists, 28 (45\%) were old age psychiatrists and 1 (2\%) was a psychiatrist in forensic learning disability.

Table 1 summarises the information respondents gave to patients about the possible effect of ECT on memory. A section of the questionnaire asking for any additional comments regarding information given on memory loss was completed by 22 of the 62 respondents. Some of the themes were as follows: seven mentioned autobiographical memory loss, four qualified that memory loss was usually temporary but might be permanent, three referred to the effects of the depression on memory and two mentioned to patients that more research was needed.

Of the 62 respondents, 33 answered a question asking them to give a brief outline if they had encountered a patient who had memory loss for longer than the period spanning ECT. Of these 33 psychiatrists, 8 commented that they had not encountered such a patient. Twenty gave outlines, examples of which are given below.

'One person lost memories for events during a foreign holiday the previous year although she could recall being on the plane coming back.'

'Several patients complained of losing "blocks" of long-term memory, e.g. childhood.'

\footnotetext{
'I have seen many people who have complained of "memory loss" despite tests showing an actual improvement in cognition after ECT. A small proportion suffer autobiographical lacunae, which doesn't seem to be easily demonstrated on testing.'
}

'Loss of discrete episodes of memory for events, e.g. holidays, family, weddings, etc. But patient considered benefits of ECT for him outweighed these negative effects.'

Table 2 shows the respondents' practices regarding the routine assessment of memory for patients undergoing ECT. Several respondents commented that they tried to follow up patients to test memory at 3 months and 6 months but this was difficult to do, and one said the referring consultant and team did this.

Of the 62 respondents, 55 left comments when asked how they assessed memory. Thirty-eight mentioned using the Mini-Mental State Examination (MMSE) or some part of it; ${ }^{11}$ however, six stated that the MMSE as a tool was insufficient and an additional four referred to having no suitable tool with which to assess memory. Thirty referred to taking into account patients' experience of memory loss if the patient complained directly, or by asking the patient directly or taking a history and doing a mental state examination. Three said they would carry out further tests if the patient complained of memory loss, including using the Addenbrooke's Cognitive Examination, ${ }^{12}$ or by referral for further neuropsychological assessment. Two were involved in research on this topic using a 'battery of tests'. original papers 
Table 2. Responses to the question 'When do you or a member of your team routinely assess memory?'

original

papers

Number answering yes/total

answering question

$n / N$

Before treatment with

$55 / 61$

90

ECT

Regularly during the

$54 / 59$

treatment course

Three months after the

$30 / 55$

55

course of ECT

Six months after the

$25 / 56$

45

course of ECT

$E C T$, electroconvulsive therapy.

Table 3 shows responses to the question, 'Who obtains informed consent from patients for ECT in your team?'. Respondents could answer 'yes' or 'no' to a list of possible staff. Not all respondents completed all questions. The 'other' category included associate specialists or doctors with Section 12 approval. Thus, more than one grade of medical professional in each service could obtain consent. It is clear that the majority of respondents considered it was the responsibility of the referring consultant to obtain consent, rather than the consultant with specialist responsibility for administering ECT.

\section{Discussion}

Almost all the ECT consultant psychiatrists in our study informed patients that ECT may cause memory loss that is temporary, permanent or for events just before and after the treatment, and also gave patients a fact sheet including information on possible memory loss. This indicates a consensus in this area. However, $79 \%$ of these psychiatrists informed patients about the possibility of longer retrograde amnesia, and less than a third informed patients about the risk of memory loss for events occurring more than 3 weeks after ECT. The findings appear to

Table 3. Responses to the question 'Who obtains informed consent from patients for ECT in your team?'

\begin{tabular}{|lcr}
\hline & $\begin{array}{c}\text { Number answering } \\
\text { yes/total } \\
\text { answering question } \\
n / N\end{array}$ & $\%$ \\
\hline Referring consultant & $59 / 59$ & 100 \\
Specialist registrar & $22 / 32$ & 69 \\
Staff grade doctor & $18 / 30$ & 60 \\
Senior house officer & $13 / 29$ & 45 \\
Specialist ECT consultant & $11 / 33$ & 33 \\
Other & $4 / 12$ & 33 \\
\hline
\end{tabular}

ECT, electroconvulsive therapy. be consistent with what is currently known about the effect of ECT on memory.

Although the majority of respondents routinely assessed memory before and during the course of ECT, memory was less likely to be assessed 3 months later and even less likely 6 months later. The most common method used was the MMSE (which a number of respondents said was inadequate for this purpose) and clinical interview.

The findings seem to reflect the lack of clarity on the best way to measure and monitor the memory side-effects of ECT. The Royal College of Psychiatrists guidance and ECTAS standards recommend that memory is assessed before and after the first ECT and reassessed at intervals throughout the treatment course, but the means of assessment and intervals are not specified. ${ }^{13,14}$

Two ECTAS standards that an accredited clinic would be expected to meet include the recording of subjective and objective cognitive side-effects between treatment sessions and a clinical interview at the end of the ECT course to establish any autobiographical memory loss. Standards for an 'excellent' clinic include recording patients' subjective and objective cognitive side-effects 3 months and 6 months after completion of a treatment course. ${ }^{14}$ The guidelines suggest use of a memory log, although details are not specified.

The referring consultant rather than the ECT consultant psychiatrist was most likely to obtain consent from the patient, and in some cases middle-grade or even junior doctors were doing this. The ECT consultant psychiatrists are unlikely to be imparting information (and their specialist knowledge of ECT) other than to their own patients. This reflects the broad guidance given by the Royal College of Psychiatrists and the ECTAS standards. The College recommends that consent is obtained only by a registered medical practitioner with adequate knowledge of the nature and effects of ECT but does not state the grade or seniority of the psychiatrist. Each ECT clinic should have a policy about who is deemed competent to obtain consent in clinical teams who refer to the clinic. ${ }^{13}$ The ECTAS standards state that the referring consultant should assess whether the patient can give valid consent. ${ }^{14}$

\section{Strengths and weaknesses}

This study has the strengths of including a national multicentre sample of ECT consultant psychiatrists, with a high response rate. However, it only included psychiatrists working at ECT clinics that had elected to participate in the ECTAS accreditation process and so they may not be representative of all ECT consultant psychiatrists. Furthermore, this study cannot show actual clinical practice and instead reports expert opinion.

\section{Implications and conclusions}

We have shown that there is a consensus of clinical practice to inform patients that ECT may cause permanent memory loss and retrograde amnesia for events just before the procedure. Most ECT consultant psychiatrists 
inform patients about retrograde amnesia for distant past events but only a minority inform them about anterograde amnesia for events occurring after the course of ECT. It is also routine practice to assess memory before, during and immediately after ECT. However, smaller numbers of patients have their memory assessed at longterm follow-up. There is consensus on the use of the MMSE despite realisation of its inadequacy.

Further research is needed into the effects of ECT on memory and the most suitable ways to assess it. Audit projects could use case notes to assess clinical practice, focusing on the type of memory effects explained to consenting patients and the routine assessment of memory at follow-up. Use of memory assessment tools other than the MMSE needs to be encouraged, including an assessment of autobiographical memory.

The referring consultant is usually the person who obtains consent for ECT from the patient, as opposed to the ECT consultant psychiatrist. It has been shown previously that a third of referring consultants find it difficult to communicate with patients about the risks of long-term cognitive effects and some did not think it appropriate to refer to memory loss as being permanent. ${ }^{8}$ It is thus important that accurate information is disseminated to referring consultants via further training. Alternatively, the ECT consultant psychiatrist could obtain consent from the patient after the referring consultant has agreed that the patient has capacity to consent. Most radically, ECT could be introduced as a specific accreditation or subspecialty in higher psychiatric training, with the ECT consultant psychiatrist becoming more involved in the consent process.

\section{Acknowledgements}

We thank all the psychiatrists who kindly completed the questionnaire and Lauren Rayner for her help. We also thank Joanne Cresswell for her helpful comments on an earlier draft and the ECTAS team for their assistance.

\section{Declaration of interest}

All the authors work with ECTAS.

\section{References}

1 The UK ECT Review Group. Efficacy and safety of electroconvulsive therapy in depressive disorders: a systematic review and metaanalysis. Lancet 2003; 361: 799-808.

2 Rose D, Fleischmann P, WykesT, Leese M, Bindman J. Patients' perspectives on electroconvulsiv therapy: systematic review. BMJ 2003; 326: 1363-7.

3 Ingram A, Saling M, Schweitzer I. electroconvulsive therapy: a review. J ECT 2008; 24: 3-9.

4 Fraser $\mathrm{LM}, \mathrm{O}^{\prime}$ Carroll RE, Ebmeier KP. The effect of electroconvulsive therapy on autobiographical memory: a systematic review. J ECT 2008; 24: 10-7.

5 Sackeim HA, Prudic J, Fuller $R$, Keilp J, Lavori PW, Olfson M. The cognitive effects of electroconvulsive therapy in community settings. Neuropsychopharmacology 2007; 32: 244-54.

6 Rose DS, WykesTH, Bindman JP, Fleischmann PS. Information, consent and perceived coercion: patients' perspectives on electroconvulsive therapy. $\mathrm{Br} J$ Psychiatry 2005; 186: 54-9.

7 Rayner L, Kershaw K, Hanna D, Chaplin R. The patient perspective of the consent process and side effects of electroconvulsive therapy. J Mental Health 2009, in press.

8 Blaj A, Worrall A, Chaplin R. Electroconvulsive therapy: the practice and training needs of referring psychiatrists in the United Cognitive side effects of brief pulse

Kingdom and Republic of Ireland. I ECT 2007; 23: 78-81.

9 Department of Health.

ElectroconvulsiveTherapy: Survey Covering Period from January 2002 to March 2002, England. Stat Bull 2003 (http://www.dh.gov.uk/ prod_consum.dh/groups/ dh.digitalassets/@dh/@en/ documents/digitalasset/ dh.4023559.pdf).

10 Cresswell J, Hood C, Lelliott P. ECTAS Second National Report October 2005-October 2007. ECTAS, 2007 (http:// www.rcpsych.ac.uk/PDF/ Ectas\%20Report\%202.pdf).

11 Folstein MF, Folstein SE, McHugh PR. 'Mini-mental state'. A practica method for grading the cognitive state of patients for the clinician. J Psychiatr Res 1975; 12 : 189-98.

12 Mathuranath PS, Nestor P, Berrios GE, RakowiczW, Hodges JR. A brief cognitive test battery to differentiateAlzheimer's disease and fronto-temporal dementia. Neurology 2000; 55: 1613-20.

13 Royal College of Psychiatrists. The ECT Handbook (Second Edition): TheThird Report of the Royal College of Psychiatrists' Special Committee on ECT (Council Report CR128). Royal College of Psychiatrists, 2005

14 Cresswell J, Hood C. ECTAS Standards for the Administration of ECT(6th edn) Electroconvulsive TherapyAccreditation Service, 2008 (http://www.rcpsych.ac.uk/ pdf/ECTAS\%20Standards $\% 20$ 2008.pdf).

*Dalia Hanna Consultant in Old Age Psychiatry, Berkshire Healthcare NHS FoundationTrust, Prospect Park Hospital, Honey End Lane, Reading RG30 4EJ, email: daliahnn@yahoo.co.uk, Kerry Kershaw PhD Student, Royal College of Psychiatrists and Goldsmiths College, Royal College of Psychiatrists' Research \& Training Unit, Robert Chaplin Research Fellow, Royal College of Psychiatrists' Research \& Training Unit, and Consultant Psychiatrist, Oxfordshire \& Buckinghamshire NHS Partnership Mental Health Trust original

papers 